\title{
Features of Electromagnetic Waves Scattering by Surface Fractal Structures
}

\author{
O. Yu. Semchuk ${ }^{1}$ and M.Willander ${ }^{2}$ \\ ${ }^{1}$ Chuiko Institute of Surface Chemistry National Academy of Sciences of Ukraine, \\ ${ }^{2}$ Department of Science and Technology Linkoping University \\ 1 Ukraine \\ ${ }^{2}$ Sweden
}

\section{Introduction}

Accurate measurement of surface roughness of machined workpieces is of fundamental importance particularly in the precision engineering and manufacturing industry. This is mainly due to the more stringent demand on material quality as well as the miniaturization of product components in these industries [1-3]. For instance, in the disk drive industry, to maintain the quality of the electrical components mounted on an optical disk, the surface roughness of the disk must be accurately measured and controlled. Hence, the surface finish, normally expressed in terms of surface roughness, is a critical parameter used for the acceptance or rejection of a product.

Surface roughness is usually determined by a mechanical stylus profilometer. However, the stylus technique has certain limitations: the mechanical contact between the stylus and the object can cause deformations or damage on the specimen surface and it is a pointwise measurement method and is time consuming. Hence a noncontact and more speed optical method would be attractive. Different optical noncontact methods for measuring surface roughness have been developed mainly based on reflected light detection, focus error detection, laser scattering, speckle and the interference method [4-10]. Some of these have good resolutions and are being applied in some sectors where mechanical measuring methods previously enjoyed clear predominance. Among these methods, the light scattering method [11] which is a noncontact area-averaging technique, is potentially more speedy for surface inspection than other profiling techniques particularly the traditional stylus technique. Other commercially available products such as the scanning tunneling microscope (STM), the atomic force microscope (AFM) and subwavelength photoresist gratings [12-15], which are pointwise techniques, are used mainly for optically smooth surfaces with roughnesses in the nanometer range.

In this chapter in the frame of the Kirchhoff method (scalar model) the average coefficient of light scattering by surface fractal structures was calculated. A normalized band-limited Weierstrass function is presented for modeling $2 \mathrm{D}$ fractal rough surfaces. On the basis of numerical calculation of average scattering coefficient the scattering indicatrises diagrams for various surfaces and falling angles were calculated. The analysis of the diagrams results in the following conclusions: the scattering is symmetrically concerning a plane of fall; with 
increase the degree of a surface calibration the picture becomes complicated; the greatest intensity of a scattering wave is observed in a mirror direction; there are other direction in which the bursts of intensity are observed.

\section{Fractal model for two-dimensional rough surfaces}

At theoretical research of processes of electromagnetic waves scattering selfsimilar heterogeneous objects (by rough surfaces) is a necessity to use the mathematical models of dispersive objects. As a basic dispersive object we will choose a rough surface. As is generally known, she is described by the function $\mathrm{z}(\mathrm{x}, \mathrm{y})$ of rejections $\mathrm{z}$ of points of $\mathrm{M}$ of surface from a supporting plane $(x, y)$ (fig.1) and requires the direct task of relief to the surface.

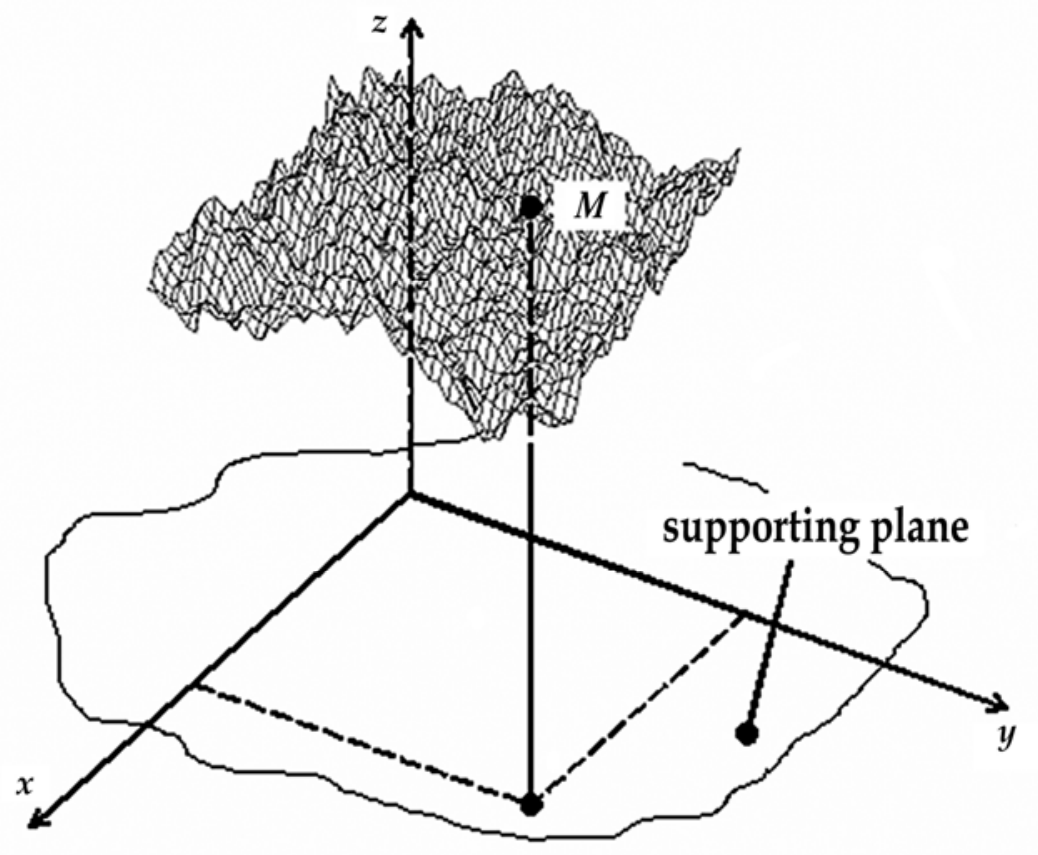

Fig. 1. Schematic image of rough surface

There are different modifications of Weierstrass-Mandelbrot function in the modern models of rough surface are used. For a design a rough surface we is used the Weierstrass limited to the stripe function $[3,4]$

$$
z(x, y)=c_{w} \sum_{n=0}^{N-1} \sum_{m=1}^{M} q^{(D-3) n} \sin \left\{K q^{n}\left[x \cos \frac{2 \pi m}{M}+y \sin \frac{2 \pi m}{M}\right]+\varphi_{n m}\right\},
$$

where $c_{w}$ is a constant which ensures that $W(x, y)$ has a unit perturbation amplitude; $q(q>1)$ is the fundamental spatial frequency; $D(2<D<3)$ is the fractal dimension; $K$ is the 
fundamental wave number; $N$ and $M$ are number of tones, and $\varphi_{n m}$ is a phase term that has a uniform distribution over the interval $[-\pi, \pi]$.

The above function is a combination of both deterministic periodic and random structures. This function is anisotropic in the two directions if $M$ and $N$ are not too large. It has a large derivative and is self similar. It is a multi-scale surface that has same roughness down to some fine scales. Since natural surfaces are generally neither purely random nor purely periodic and often anisotropic, the above proposed function is a good candidate for modeling natural surfaces.

The phases $\varphi_{n m}$ can be chosen determinedly or casually, receiving accordingly determine or stochastic function $z(x, y)$. We further shall consider $\varphi_{n m}$ as casual values, which in regular distributed on a piece $[-\pi ; \pi]$. With each particular choice of numerical meanings all $N \times M$ phases $\varphi_{n m}$ (for example, with the help of the generator of random numbers) we receive particular (with the beforehand chosen meanings of parameters $c_{w}, q, K, D, N, M$ ) realization of function $z(x, y)$. The every possible realizations of function $z(x, y)$ form ensemble of surfaces.

A deviation of points of a rough surface from a basic plane proportional $c_{w}$, therefore this parameter is connected to height of inequalities of a structure of a surface. Further it is found to set a rough surface, specifying root-mean-square height of its structure $\sigma$, which is determined by such grade:

$$
\sigma \equiv \sqrt{\left\langle h^{2}\right\rangle}
$$

where $h=z(x, y),\langle\ldots\rangle=\prod_{n=0}^{N-1} \prod_{m=1}^{M} \int_{-\pi}^{\pi} \frac{d \varphi_{n m}}{2 \pi}(\ldots)$ - averaging on ensemble of surfaces.

The connection between $c_{w}$ and $\sigma$ can be established, directly calculating integrals:

$$
\sigma=\left[\prod_{n=0}^{N-1} \prod_{m=1}^{M} \int_{-\pi}^{\pi} \frac{d \varphi_{n m}}{2 \pi} z^{2}(x, y)\right]^{\frac{1}{2}}=c_{w}\left[\frac{M\left(1-q^{2 N(D-3)}\right)}{2\left(1-q^{2(D-3)}\right)}\right]^{\frac{1}{2}} .
$$

So, the rough surface in our model is described by function from six parameters: $c_{w}$ (or ), $q, K, D, N, M$. The influence of different parameters on a kind of a surface can be investigated analytically, and also studying structures of surfaces constructed by results of numerical accounts of Weierstrass function. Analysis of the surface profiles built by us on results of numeral calculations (fig. 2) due to the next conclusions:

- the wave number $K$ sets length of a wave of the basic harmonic of a surface;

- the numbers $N, M, D$ and $q$ determine a degree of a surface calibration at the expense of imposing on the basic wave from additional harmonics, and $N$ and $M$ determine the number of harmonics, which are imposed;

- $\quad D$ determines amplitude of harmonics;

- $\quad q$ - both amplitude, and frequency of harmonics.

Let's notice that with increase $N, M, D$ and $q$ the spatial uniformity of a surface on a large scale is increased also. 

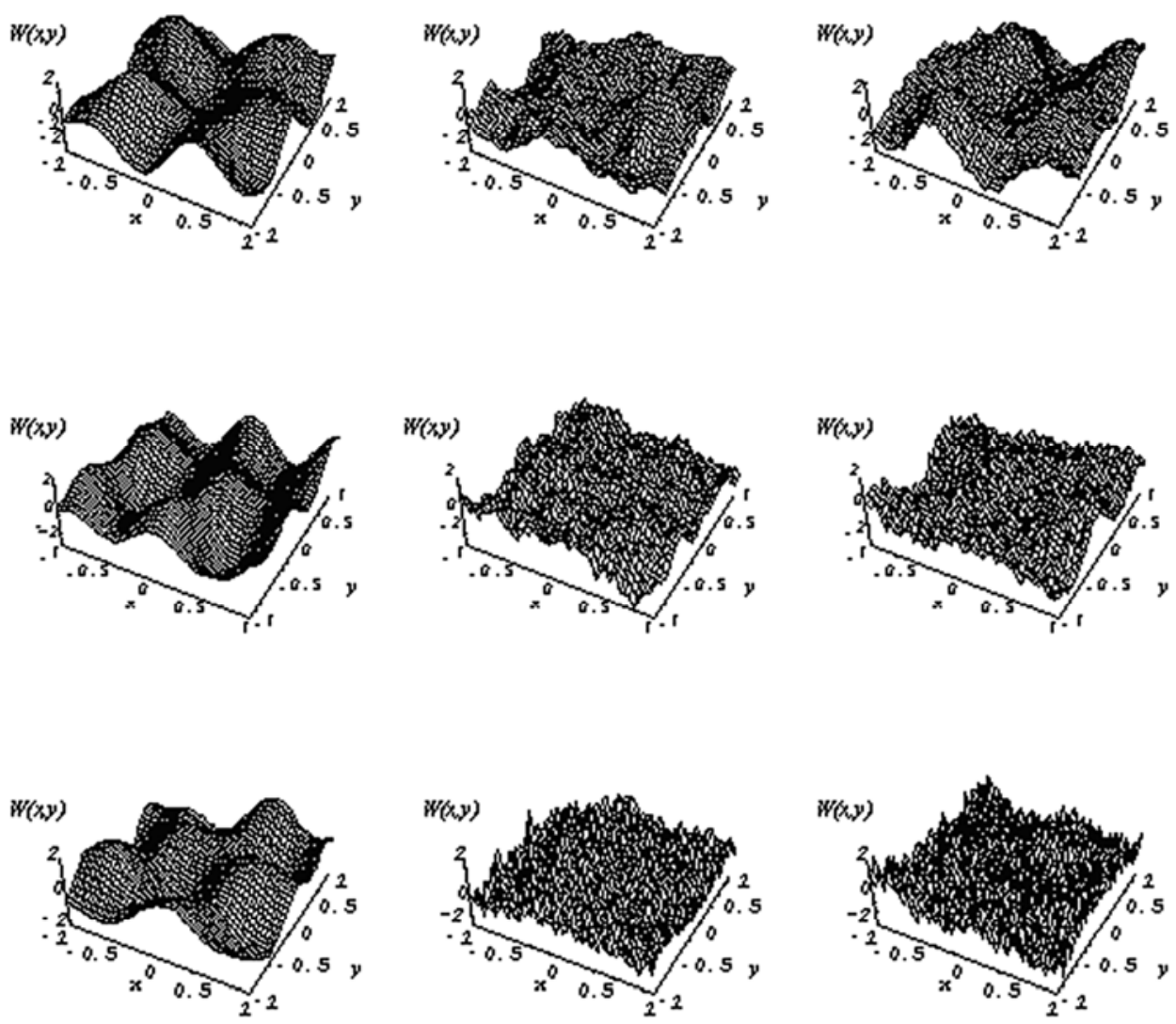

Fig. 2. Examples of rough surface by the Weierstrass function $K=2 \pi ; N=M=5 ; \sigma=1$. $D=2,1 ; D=2,5 ; D=2,9$ (from above to the bottom) $q=1,1 ; q=3 ; q=7$ (from left to right)

By means of the original program worked out by us in the environment of Mathematika 5.1 there was the created base of these various types of fractal dispersive surfaces on the basis of Weierstrass function.

Influence each of parameters $q, K, D, N, M$ on character of profile of surface it appears difficult enough and determined by values all other parameters. So, for example, at a value $D=2,1$, what near to minimum $(D=2)$, the increase of size q does not almost change the type of surface (see the first column on fig.2). With the increase of size D the profile of surface becomes more sensible to the value $q$ (see the second and third columns on fig.2). Will notice that with an increase $N, M, D$ and $\mathrm{q}$ increases and spatial homogeneity of surface on grand the scale: large-scale "hills" disappear, and finely scale heterogeneities remind a more mesentery on a flat surface. 


\section{Electromagnetic wave scattering on surface fractal structures}

At falling of electromagnetic wave there is her dispersion on the area of rough surface - the removed wave scattering not only in direction of floppy, and, in general speaking, in different directions. Intensity of the radiation dissipated in that or other direction is determined by both parameters actually surfaces (by a reflectivity, in high, by a form and character of location of inequalities) and parameters of falling wave (frequency, polarization) and parameters of geometry of experiment (corner of falling). The task of this subdivision is establishing a connection between intensity of the light dissipated by a fractal surface in that or other direction, and parameters of surface.

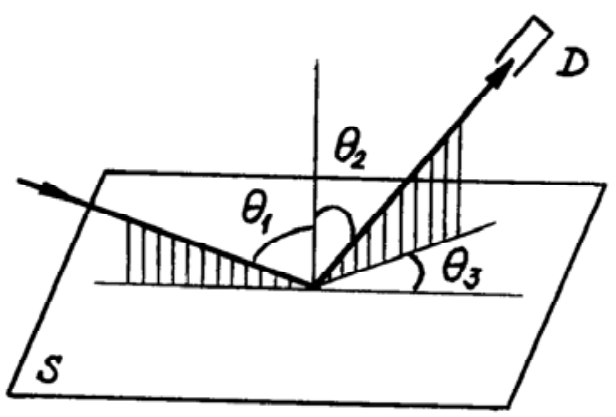

Fig. 3. The scheme of experiment on light scattering by fractal surface: $\mathrm{S}$ is a scattering surface; D-detector, $\theta_{1}$ is a falling angle; $\theta_{2}$ is a polar angle; $\theta_{3}$ is an azimuthally angle

The initial light wave falls on a rough surface $S$ under a angle $\theta_{1}$ and scattering in all directions. The scattering wave is observed by means of the detector $\mathrm{D}$ in a direction which is characterized by a polar angle $\theta_{2}$ and an azimuthally angle $\theta_{3}$. The measured size is intensity of light $I_{s}$ scattered at a direction $\left(\theta_{2}, \theta_{3}\right)$. Our purpose is construction scattering indicatrise of an electromagnetic wave by a fractal surface (1).

As $I_{s}=\vec{E}_{s} \cdot \vec{E}_{s}^{*}$ (where $\vec{E}_{s}$ is an electric field of the scattering wave in complex representation) that the problem of a finding $I_{s}$ is reduced to a finding of the scattered field $\vec{E}_{s}$.

The scattered field we shall find behind Kirchhoff method [16], and considering complexity of a problem, we shall take advantage of more simple scalar variant of the theory according to which the electromagnetic field is described by scalar size. Thus we lose an opportunity to analyze polarizing effects

The base formula of a Kirchhoff method allows to find the scattered field under such conditions:

- $\quad$ the falling wave is monochromatic and plane;

- a scattered surface rough inside of some rectangular $\left(-\mathrm{X}<\mathrm{x}_{0}<\mathrm{X},-\mathrm{Y}<\mathrm{y}_{0}<\mathrm{Y}\right)$ and corpulent outside of its borders;

- $\quad$ the size of a rough site much greater for length of a falling wave;

- all points of a surface have the ended gradient;

- $\quad$ the reflection coefficient identical to all points of a surface; 
- the scattering field is observed in a wave zone, i.e. is far enough from a scattering surface.

Under these conditions the scattered field is given by

$$
E_{s}(\vec{r})=-i k r F\left(\theta_{1}, \theta_{2}, \theta_{3}\right) \frac{\exp (i k r)}{2 \pi r} \int_{S_{0}} \exp \left[i k \varphi\left(x_{0}, y_{0}\right)\right] d x_{0} d y_{0}+E_{e}(\vec{r}),
$$

Where $k$ is the wave number of falling wave; $F\left(\theta_{1}, \theta_{2}, \theta_{3}\right)=-\frac{R}{2 C}\left(A^{2}+B^{2}+C^{2}\right)$ is a angle factor; $\mathrm{R}$ - scattering coefficient; $\varphi\left(\mathrm{x}_{0}, \mathrm{y}_{0}\right)=\mathrm{Ax} \mathrm{x}_{0}+\mathrm{By_{0 }}+\mathrm{Ch}\left(\mathrm{x}_{0}, \mathrm{y}_{0}\right)$ is the phase function; $\mathrm{h}\left(\mathrm{x}_{0}, \mathrm{y}_{0}\right)=\mathrm{z}\left(\mathrm{x}_{0}, \mathrm{y}_{0}\right) ; \quad \mathrm{A}=\sin \theta_{1}-\sin \theta_{2} \cos \theta_{3} ; \quad \mathrm{B}=-\sin \theta_{2} \sin \theta_{3} ; \quad \mathrm{C}=-\cos \theta_{1}-\cos \theta_{2} ;$ $\mathrm{E}_{\mathrm{e}}(\overrightarrow{\mathrm{r}})=-\frac{\mathrm{R}}{\mathrm{C}} \cdot \frac{\exp (\mathrm{ikr})}{4 \pi \mathrm{r}}\left(\mathrm{AI}_{1}+\mathrm{BI}_{2}\right)$,

$$
\begin{aligned}
& I_{1}=\int_{-Y}^{Y}\left[e^{i k \varphi\left(X, y_{0}\right)}-e^{i k \varphi\left(-X, y_{0}\right)}\right] d y_{0}, \\
& I_{2}=\int_{-X}^{X}\left[e^{i k \varphi\left(x_{0}, Y\right)}-e^{i k \varphi\left(x_{0},-Y\right)}\right] d x_{0} .
\end{aligned}
$$

After calculation of integrals (4) and (5) by means of the formula

$$
\mathrm{e}^{\mathrm{i} z \sin \phi}=\sum_{1=-\infty}^{\infty} \mathrm{I}_{1}(\mathrm{z}) \mathrm{e}^{\mathrm{i} l \phi}
$$

(where $\mathrm{I}_{1}(\mathrm{z})$ is the Bessel function of the whole order), we receive

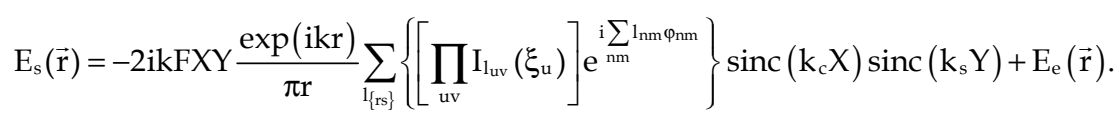

where

$$
\begin{aligned}
& F=F\left(\theta_{1}, \theta_{2}, \theta_{3}\right), \sum_{\left.1_{\{\mathrm{rs}}\right\}} \equiv \sum_{1_{0,1}=-\infty}^{\infty} \sum_{1_{0,2}=-\infty}^{\infty} \ldots \sum_{1_{(\mathrm{N}-1), \mathrm{M}=-\infty}^{\infty},}^{\infty} \prod_{\mathrm{uv}} \equiv \prod_{\mathrm{u}=1}^{\mathrm{N}-1} \prod_{\mathrm{v}=0}^{\mathrm{M}}, \quad \sum_{\mathrm{nm}} \equiv \sum_{\mathrm{n}=1}^{\mathrm{N}-1} \sum_{\mathrm{m}=0}^{\mathrm{M}}, \\
& \xi_{\mathrm{u}} \equiv \mathrm{kc}_{\mathrm{w}} \mathrm{Cq} \mathrm{q}^{(\mathrm{D}-3) \mathrm{u}}, \operatorname{sinc} \mathrm{x} \equiv \frac{\sin \mathrm{x}}{\mathrm{x}}, \begin{array}{l}
\mathrm{k}_{\mathrm{c}} \equiv \mathrm{kA}+\mathrm{K} \sum_{\mathrm{nm}} \mathrm{q}^{\mathrm{n}} \mathrm{l}_{\mathrm{nm}} \cos \frac{2 \pi \mathrm{m}}{\mathrm{M}} \\
\mathrm{k}_{\mathrm{s}} \equiv \mathrm{kB}+\mathrm{K} \sum_{\mathrm{nm}} \mathrm{q}^{\mathrm{n}} \mathrm{l}_{\mathrm{nm}} \sin \frac{2 \pi \mathrm{m}}{\mathrm{M}},
\end{array} \\
& E_{e}(\vec{r})=-i k X Y \frac{R}{C}\left(A^{2}+B^{2}\right) \frac{e^{i k r}}{\pi r} \operatorname{sinc}(k A X) \operatorname{sinc}(k B Y) .
\end{aligned}
$$

Thus, expression (6) gives the decision of a problem about finding a field scattering by a fractal surface, within the limits of Kirchhoff method. 
Now under the formula (4) it is possible to calculate intensity of scattered waves if to set parameters of a disseminating surface $c_{w}$ (or) $\sigma D, q, K, N, M, X, Y, \phi_{n m}$, parameter $k$ (or $\left.\lambda=\frac{2 \pi}{k}\right)$ a falling wave and parameters $\theta_{1}, \theta_{2}, \theta_{3}$ of geometry of experiment. This intensity will be to characterize scattering on concrete realization of a surface $z(x, y)$ (with a concrete set of casual phases $\phi_{n m}$ ). For comparison of calculations with experimental data it is necessary to operate with average on ensemble of surfaces intensity $\left\langle I_{s}\right\rangle=\left\langle\vec{E}_{s} \vec{E}_{s}^{*}\right\rangle$. Such intensity has appeared proportional intensity $I_{0}=\left(\frac{2 k X Y \cos \Theta_{1}}{\pi r}\right)^{2}$ of the wave reflected from the corresponding smooth basic surface, therefore for the theoretical analysis of results it is more convenient to use average scattering coefficient

$$
\left\langle\rho_{s}\right\rangle=\frac{\left\langle I_{s}\right\rangle}{I_{0}} .
$$

After calculation $\left\langle I_{s}\right\rangle$ and leaving from (6), we shall receive exact expression

$$
\begin{gathered}
\left\langle\rho_{s}\right\rangle=\left[\frac{F\left(\theta_{1}, \theta_{2}, \theta_{3}\right)}{\cos \Theta_{1}}\right]^{2} \sum_{\{r s\}}\left\{\prod_{u v} I_{l u v}^{2}\left(\xi_{u}\right) \operatorname{sinc}^{2}\left(\mathrm{k}_{\mathrm{c}} \mathrm{X}\right) \operatorname{sinc}^{2}\left(\mathrm{k}_{\mathrm{s}} \mathrm{Y}\right)\right\}+ \\
+\left[\frac{R\left(A^{2}+B^{2}\right)}{2 C \cos \theta_{1}}\right]^{2} \operatorname{sinc}^{2}(\mathrm{kAX}) \operatorname{sinc}^{2}(\mathrm{kBY})
\end{gathered}
$$

As expression (7) consist the infinite sum to use it for numerical calculations inconveniently. Essential simplification is reached in case of $\xi_{\mathrm{n}}<1$. Using thus decomposition function in a line

$$
I_{v}(z)=\left(\frac{3}{2}\right)^{v} \sum_{k=0}^{\infty} \frac{\left(-z^{2} / 4\right)^{k}}{k ! \Gamma(v+k+1)},
$$

that rejecting members of orders, greater than $\xi_{n}^{2}$. We shall receive the approached expression for average scattering coefficient

$$
\begin{gathered}
\left\langle\rho_{s}\right\rangle \approx\left[\frac{F\left(\theta_{1}, \theta_{2}, \theta_{3}\right)}{\cos \theta_{1}}\right]^{2}\left\{\left[1-(k \sigma C)^{2}\right] \operatorname{sinc}^{2}(k A X) \operatorname{sinc}^{2}(k B Y)+\right. \\
\left.+\frac{1}{2} c_{\mathrm{f}}^{2} \sum_{\mathrm{nm}} \mathrm{q}^{2(\mathrm{D}-3) \mathrm{n}} \operatorname{sinc}^{2}\left[\left(\mathrm{kA}+\mathrm{Kq}^{\mathrm{n}} \cos \frac{2 \pi \mathrm{m}}{\mathrm{M}}\right) \mathrm{X}\right] \operatorname{sinc}^{2}\left[\left(\mathrm{kB}+\mathrm{Kq}^{\mathrm{n}} \sin \frac{2 \pi \mathrm{m}}{\mathrm{M}}\right) \mathrm{Y}\right]\right\}+ \\
+\left[\frac{\mathrm{R}}{2 C \cos \theta_{1}}\left(A^{2}+B^{2}\right)\right]^{2} \operatorname{sinc}^{2}(\mathrm{kAX}) \operatorname{sinc}^{2}(\mathrm{kBY})
\end{gathered}
$$

where 


$$
\mathrm{c}_{\mathrm{f}} \equiv \mathrm{kc}_{\mathrm{W}} \mathrm{C}=\mathrm{k} \sigma \mathrm{C}\left[\frac{2}{\mathrm{M}} \cdot \frac{1-\mathrm{q}^{2(\mathrm{D}-3)}}{1-\mathrm{q}^{2 \mathrm{~N}(\mathrm{D}-3)}}\right]^{\frac{1}{2}}
$$

\section{Results of numerical calculations}

On the basis of numerical calculations of average factor of dispersion under the formula (8) we had been constructed the average scattering coefficient $\left\langle\rho_{\mathrm{s}}\right\rangle$ from $\theta_{2}$ and $\theta_{3}$. (scattering indicatrix diagrams) for different types of scattering surfaces. At the calculations we have supposed $R=1$, and consequently did not consider real dependence of reflection coefficient $\mathrm{R}$ from the length of a falling wave $\lambda$ and a falling angle $\theta_{1}$. The received results are presented on fig. 4.

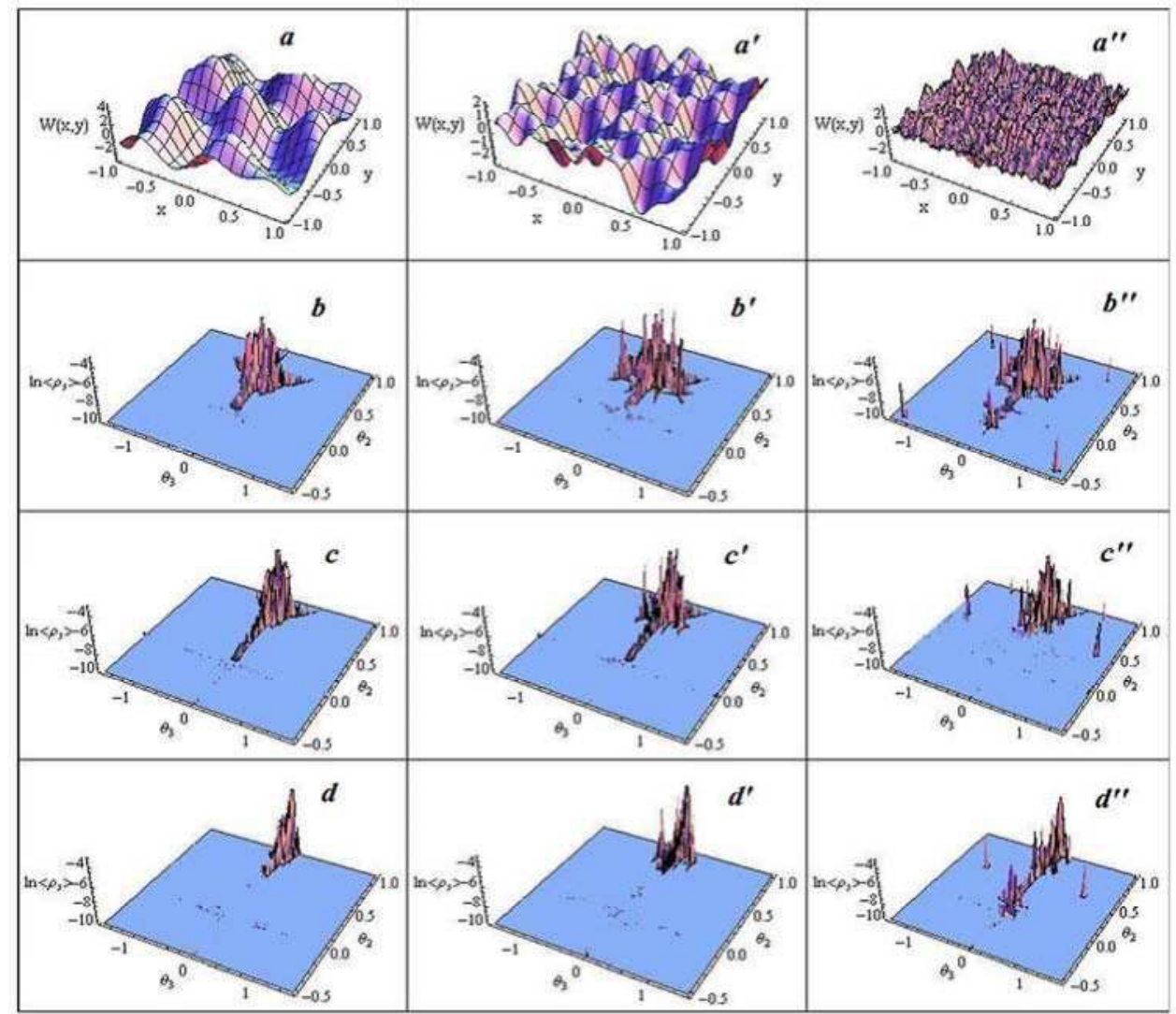

Fig. 4. Dependencies of the $\log \left\langle\rho_{s}\right\rangle$ from the angles $\theta_{2}$ and $\theta_{3}$ for the various type of fractal surfaces: $a, a^{\prime}, a^{\prime \prime}$ - the samples of rough surfaces, which the calculation of dispersion indexes was produced; from top to bottom the change of scattering index is rotined for three angles of incidence $\theta_{1}=30,40,60^{\circ}\left(a-d, a^{\prime}-d^{\prime}, a^{\prime \prime}-d^{\prime \prime}\right)$ at $\mathrm{N}=5, \mathrm{M}=10, \mathrm{D}=2.9, \mathrm{q}=1.1 ; \mathrm{n}=2, \mathrm{M}=3$, $\mathrm{D}=2.5, \mathrm{q}=3 ; \mathrm{N}=5, \mathrm{M}=10, \mathrm{D} .2 .5, \mathrm{q}=3$ accordingly 
The analysis of schedules leads to such results:

- Scattering is symmetric concerning of a falling plane;

- The greatest intensity of the scattering wave is observed in a direction of mirror reflection;

- $\quad$ There are other directions in which splashes in intensity are observed;

- With increase in a calibration degree of surfaces (or with growth of its large-scale heterogeneity) the picture of scattering becomes complicated. Independence of the type of scattering surface there is dependence of the scattering coefficient from the incidence angle of light wave. As far as an increase of the incidence angle from $30^{\circ}$ to $60^{\circ}$ amounts of additional peaks diminishes. Is their most number observed at $\theta_{1}=30^{\circ}$. It is related to influence on the scattering process of the height of heterogeneity of the surface. At the increase of the angle of incidence of the falling light begins as though not to "notice" the height of non heterogeneity and deposit from them diminishes.

The noted features of dispersion are investigation of combination of chaoticness and selfsimilarity relief of scattering surface.

\section{Conclusion}

In this chapter in the frame of the Kirchhoff method the average coefficient of light scattering by surface fractal structures was calculated. A normalized band-limited Weierstrass function is presented for modeling 2D fractal rough surfaces. On the basis of numerical calculation of average scattering coefficient the scattering indicatrises diagrams for various surfaces and falling angles were calculated. The analysis of the diagrams results in the following conclusions: the scattering is symmetrically concerning a plane of fall; with increase the degree of a surface calibration the picture becomes complicated; the greatest intensity of a scattering wave is observed in a mirror direction; there are other direction in which the bursts of intensity are observed.

\section{References}

[1] Bifano, T. G. Fawcett, H. E. \& Bierden, P. A. (1997). Precision manufacture of optical disc master stampers. Precis. Eng. 20(1), 53-62.

[2] Wilkinson, P. et al. (1997). Surface finish parameters as diagnostics of tool wear in face milling, Wear 25(1), 47-54.

[3] Sherrington, I.' \& Smith, E. H. (1986). The significance of surface topography in engineering. Precis. Eng. 8(2). 79-87.

[4] Kaneami, J. \& Hatazawa, T. (1989). Measurement of surface profiles bv the focus method. Wear 134, 221-229.

[5] Mitsui, K. (1986). In-process sensors for surface roughness and their applications. Precis. Eng. 8(40), 212-220.

[6] Baumgart, J. W. \& Truckenbrodt H. (1998). Scatterometrv of honed surfaces. Opt. Eng. 37(5), 1435-1441.

[7] Tay, C. J. Toh, L S., Shang, H. M. \& Zhang, J. B. (1995). Whole-field determination of surface roughness bv speckle correlation. Appl. Opt. 34(13). 2324-2335.

[8] Peiponen, K. E. \& Tsuboi, T. (1990). Metal surface roughness and optical reflectance Opt. Laser Technol. 22(2). 127-130. 
[9] Whitley, J. Q., Kusy, R. P., Mayhew, M. J. \& Buckthat, J. E. (1987). Surface roughness of stainless steel and electroformed nickle standards using HeNe laser. Opt. Laser Technol 19(4), 189-196.

[10] Mitsui, M., Sakai, A. \& Kizuka, O. (1988'). Development of a high resolution sensor for surface roughness, Opt. Eng. 27(^6). 498-502.

[11] Vorburger, T. V., Marx, E. \& Lettieri, T. R. (1993). Regimes of surface roughness measurable with light scattering. Appl Opt. 32(19!. 3401-3408.

[12] Raymond, C. J., Murnane, M. R., H. Naqvi, S. S. \& Mcneil J. R. (1995). Metrology of subwavelength photoresist gratings usine optical scat-terometry. J. Vac. Sci. Technol B 13(4), 1484-1495.

[13] Whitehouse, D. J. (1991). Nanotechnologv instrumentation. Meas. Control 24(3), 37-46.

[14] Madsen, L. L, J. Srgensen,J. F., Carneiro, K. \& Nielsen, H. S. (1993-1994). Roughness of smooth surfaces: STM versus profilometers. Metro-logia 30. 513-516.

[15] Stedman, M. (1992). The performance and limits of scanning probe microscopes. In Proc. Int. Congr. X-ray Optics and Microanalysis, pp. 347-352, Manchester. IOP Publishing Ltd.

[16] Berry, M.V. \& Levis Z.V. (1980). On the Weirstrass-Mandelbrot fractal function. Proc.Royal Soc. London A, v.370, p.459. 


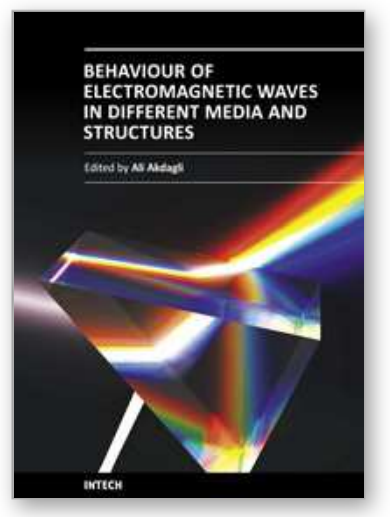

\author{
Behaviour of Electromagnetic Waves in Different Media and \\ Structures \\ Edited by Prof. Ali Akdagli
}

ISBN 978-953-307-302-6

Hard cover, 440 pages

Publisher InTech

Published online 09, June, 2011

Published in print edition June, 2011

This comprehensive volume thoroughly covers wave propagation behaviors and computational techniques for electromagnetic waves in different complex media. The chapter authors describe powerful and sophisticated analytic and numerical methods to solve their specific electromagnetic problems for complex media and geometries as well. This book will be of interest to electromagnetics and microwave engineers, physicists and scientists.

\title{
How to reference
}

In order to correctly reference this scholarly work, feel free to copy and paste the following:

O. Yu. Semchuk and M. Willander (2011). Features of Electromagnetic Waves Scattering by Surface Fractal Structures, Behaviour of Electromagnetic Waves in Different Media and Structures, Prof. Ali Akdagli (Ed.), ISBN: 978-953-307-302-6, InTech, Available from: http://www.intechopen.com/books/behavior-ofelectromagnetic-waves-in-different-media-and-structures/features-of-electromagnetic-waves-scattering-bysurface-fractal-structures 1

\section{INTECH}

open science | open minds

\section{InTech Europe}

University Campus STeP Ri

Slavka Krautzeka 83/A

51000 Rijeka, Croatia

Phone: +385 (51) 770447

Fax: +385 (51) 686166

www.intechopen.com

\section{InTech China}

Unit 405, Office Block, Hotel Equatorial Shanghai

No.65, Yan An Road (West), Shanghai, 200040, China

中国上海市延安西路 65 号上海国际贵都大饭店办公楼 405 单元

Phone: +86-21-62489820

Fax: +86-21-62489821 
(C) 2011 The Author(s). Licensee IntechOpen. This chapter is distributed under the terms of the Creative Commons Attribution-NonCommercialShareAlike-3.0 License, which permits use, distribution and reproduction for non-commercial purposes, provided the original is properly cited and derivative works building on this content are distributed under the same license. 